\title{
Quelques aspects rhéologiques des fluides colloïdaux
}

\author{
William R. Schowalter \\ Department of Chemical Engineering - Princeton University - USA
}

\section{Introduction}

Les colloïdes sont des particules dont la taille caractéristique est comprise entre $0.01 \mu \mathrm{m}$ et $10 \mu \mathrm{m}$. Leurs applications pratiques sont très anciennes et remontent aux premières civilisations ayant utilisé des encres et donné forme à des matériaux céramiques. Il n'est donc pas étonnant que, depuis plusieurs générations, la science des colloïdes ait attiré l'attention occasionnelle d'éminents spécialistes de la physique et de la chimie. Au début du siècle, une bonne compréhension, à caractère fondamental, de leur comportement a été acquise grâce à l'intérêt croissant porté sur le sujet. Ainsi, les fondements de la théorie du mouvement brownien ont été introduits et furent la source de beaucoup de réflexion illustrée notamment par les travaux d'Einstein, Smoluchowski et leurs contemporains. Quelques années plus tard l'ultracentrifugation fut perfectionnée pour permettre la séparation de particules colloïdales.

Après une génération de relatif oubli, la recherche sur les colloödes suscite à nouveau un regain d'intérêt. L'enthousiasme pour l'aspect fondamental vient de la fascination actuelle des physiciens pour les structures complexes et l'usage des fractales pour les décrire. Pourtant, de nombreuses questions fondamentales n'ont pas d'incidence sur les préoccupations d'ordre pratique associées à la technologie moderne.

L'industrie chimique souhaite aujourd'hui développer des produits (au lieu de procédés) dont la «valeur ajoutée " est importante. Parmi ceux-ci on peut citer les peintures latex, les revêtements spéciaux, les céramiques destinées aux structures, certains produits pharmaceutiques, les insecticides, les cosmétiques, etc., dont la mise en cuvre doit être rendue la plus facile possible. La valeur ajoutée de ces produits dépend autant de leurs propriétés colloïdales que de leur constitution chimique particulière. Ainsi, de nombreuses entreprises se sont aperçues qu'il est dans leur propre intérêt, et même essentiel, de connaître les forces qui agissent, au niveau microscopique, et qui seront, ainsi finalement, responsables de l'accueil réservé par le marché à une peinture ou un cosmétique.

C'est pour cela que cet exposé sera consacré essentiellement à l'influence des phénomènes microscopiques sur le comportement rhéologique global des suspensions colloïdales au cours du processus de déformation dynamique auquel elles sont soumises. Pour fixer le cadre de notre sujet, nous considérerons tout d'abord l'hydrodynamique des suspensions diluées. Le mouvement brownien et les interactions hydrodynamiques seront abordés ensuite. On montrera que les modèles qui en découlent présentent de sérieuses déficiences dues aux fortes interactions entre l'hydrodynamique et les forces entre particules, celles-ci étant dues à la nature intrinsèque des particules, aux ions absorbés sur leur surface dans un milieu aqueux, et/ou aux polymères presque toujours présents dans les suspensions colloïdales commercialisées. Plusieurs exemples extraits de recherches récentes seront utilisés pour illustrer ces problèmes. On terminera en suggérant quelques sujets de recherches.

\section{Some rheological aspects of colloidal fluids}

In the case where the interaction between particles is negligible, the hydrodynamic approach of suspension rheology is well known. In this paper, we present, after a brief review of classical results, a summary of recent methods allowing to account for the interaction between particles and for the effects due to the colloidal properties : brownian motion, electrostatic repulsion, and attraction due to van der Waals forces. Methods designed to correlate and explain the relationship between the structure of the colloidal particles and the behaviour of the suspension are described. Recent results concerning the significant influence of additives on the particle aggregation, and then on the rheology of colloids, are presented. Finally, future research topics are proposed. 


\section{Approches hydrodynamiques de la rhéologie des suspensions colloïdales}

Il est indéniable que les travaux d'EINSTEIN [1906, 1911] concernant la viscosité d'une suspension diluée de sphères dans un fluide newtonien, ont eu une influence considérable sur le développement de la rhéologie. Le modèle suivant lequel le comportement d'une suspension soumise à un cisaillement est équivalent à celui d'une sphère unique placée dans un fluide qui, avant l'introduction de la particule, est caractérisé par un taux de cisaillement constant, est plus puissant que ce qu'Einstein a pu probablement imaginer. Il calcula la dissipation visqueuse supplémentaire due à la présence de la sphère dans un fluide de viscosité $\mu_{0}$ et détermina ainsi la viscosité effective (apparente), $\mu_{s}$, d'une suspension dont la fraction volumique de particules rigides est $\phi$.

$$
\mu_{s}=\mu_{0}\left(1+\frac{5}{2} \phi\right) \text {. }
$$

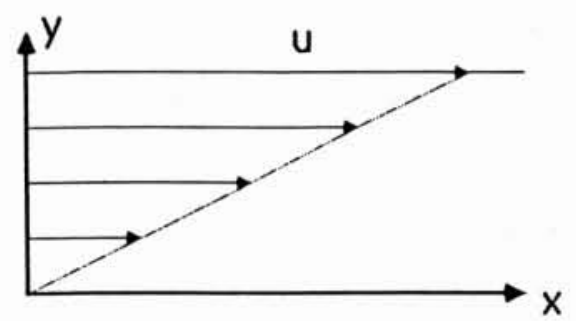

1. Ecoulement de cisaillement simple.

Bien plus de renseignements sont fournis par un calcul détaillé de l'écoulement à proximité de la sphère. Dans un écoulement de cisaillement laminaire du type,

$$
u=G y
$$

où le taux de cisaillement $G$ est constant (fig. 1), la présence de la sphère donne lieu à des lignes de courants fermées qui ont, évidemment, des conséquences importantes sur les propriétés de la suspension : coefficients de transfert de chaleur et de masse par exemple. Bien que les lignes de courant fermées soient inhérentes aux premières solutions de l'équation de Stokes, leur présence semble n'avoir été mise en évidence que beaucoup plus tard par COX, ZIA et MASSON [1968]. Ainsi, comme le montre la figure 2, chaque particule est entourée d'une certaine quantité de fluide environnant.

Une deuxième particularité de ce problème est la multiplicité de résultats disponibles quand on généralise (2) par,

$$
\mathbf{u}=\Gamma \cdot \mathbf{x}
$$

où $\Gamma$ est une constante. Bien que (1) soit indépendante de la forme de $\Gamma$, la structure de l'écoulement à proximité de la sphère en dépend fortement. Par exemple, les lignes de courant fermées de la figure 2 disparaissent si l'on considère un écoulement, non perturbé par la sphère, dans lequel les éléments fluides sont tendus (et comprimés) mais ne tournent pas. Une telle situation correspond à,

$$
\Gamma=G\left[\begin{array}{rrr}
1 & 0 & 0 \\
0 & -1 & 0 \\
0 & 0 & 0
\end{array}\right]
$$

et représente un écoulement élongationnel, prototype de celui à partir duquel les rhéologues mesurent la viscosité élongationnelle.

Il est intéressant de noter que si les particules ne sont pas des sphères rigides, mais asymétriques et déformables, le comportement rhéologique sera très influencé par la forme de $\Gamma$. Par exemple, les gouttes d'un liquide non miscible avec le fluide dans lequel elles sont en suspension se déformeront tout à fait différemment suivant que le champ est donné par (4) ou par (2) [HAKIMI et SCHOWALTER, 1980].

Depuis les travaux d'Einstein, l'étape la plus importante dans l'étude de la viscosité des suspensions a été l'extension, par JEFFERY [1922], des sphères aux ellipsoïdes. Les résultats sont bien plus qu'un exercice ennuyeux (bien qu'il y ait un peu de ça) dû à la perte de la symétrie sphérique. Dans un écoulement donné par (2), les ellipsoïdes tournent avec une vitesse angulaire qui varie avec leur orientation et donc avec le temps. Par exemple, dans le cas particulier des sphéroïdes (ellipsoïdes avec petits axes égaux), on aura un mouvement périodique et l'intersection du prolongement du grand axe avec une sphère centrée sur la particule décrira une «orbite de Jeffery ». Celle-ci dépendra de l'allongement de la particule et de son orientation à l'instant initial. Deux caractéristiques du mouvement ont des conséquences rhéologiques importantes. Premièrement, la vitesse angulaire d'une particule sur son orbite dépend de son allongement et de son orientation instantanée. Donc, en général, la vitesse angulaire n'est pas constante sur une orbite de Jeffery et les particules peuvent se trouver dans certaines orientations plutôt que dans d'autres. Ainsi, une suspension de sphéroïdes ayant une distribution d'orientation uniforme (où toutes les orientations sont possibles) à l'instant initial aboutira, avec le temps, à une distribution non uniforme. Ceci résulte de ce que, pendant leur rotation, les particules restent dans une certaine région de l'espace de configuration plus longtemps que dans d'autres. La rhéologie de la suspension

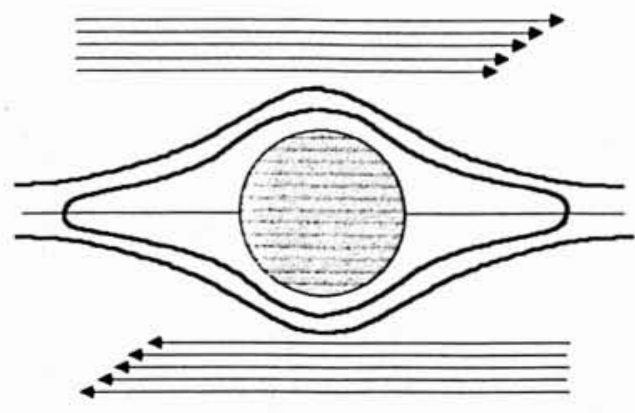

2. Lignes de courant fermées autour d'une sphère. 
dépendra donc fortement de la fonction de distribution. Dans le cas extrême d'un écoulement élongationnel (4), n'importe quelle distribution initiale (à part quelques cas singuliers) donnera lieu, après un certain temps, à un alignement des grands axes. Deuxièmement, étant donné que la rotation dépend des conditions initiales, la rhéologie des suspensions est toujours fonction de celles-ci. Ceci implique que la suspension a une mémoire infinie, ce qui est contraire à l'intuition et à la connaissance du réel. Nous reprendrons cette question plus loin.

L'étape suivante consiste à remplacer la particule rigide par une particule déformable. Une des premières approches d'une loi de comportement viscoélastique apparut à travers une application de la méthodologie d'Einstein à des particules élastiques faiblement déformables. Ceci donna lieu à une étude [FRÖHLICH et SACK, 1946] conduisant à l'établissement d'une loi de comportement du type d'Oldroyd et amena cet auteur à reconnaître ainsi la nécessité de définir des formes nouvelles des dérivées temporelles dans les lois de comportement [OLDROYD, 1950]. Pour des bulles ou des gouttes dans une phase continue liquide, de nombreuses conséquences intéressantes ont été présentées dans la littérature scientifique [RALLISON, 1980]. L'une d'elles est que la tension interfaciale agit comme une "source " d'élasticité, de sorte que dans la loi de comportement apparaissent des termes qui reflètent cette caractéristique même si les deux phases en présence sont purement visqueuses. Une autre est, comme il a déjà été mentionné, que la forme des particules en suspension dépend de la matrice de $\Gamma$. On a donc un modèle où des anisotropies sont introduites par la grandeur de la déformation et par l'orientation moyenne des gouttes ou bulles déformées.

Naturellement, il serait souhaitable de procéder à une analyse similaire pour des concentrations plus importantes de manière à inclure les effets d'interaction entre particules. Ceci est difficile. Non seulement la solution des équations appropriées devient plus problématique du fait qu'il est nécessaire de tenir compte des conditions aux limites sur la surface de nombreuses sphères, mais on doit également spécifier une distribution initiale de l'orientation des particules cohérente avec la physique du problème. Les conditions initiales appropriées ne sont pas évidentes, même pour un cas aussi simple que celui de l'écoulement de cisaillement donné par (2). Un travail de qualité sur le sujet a été réalisé par Batchelor et ses coauteurs. Pour l'écoulement élongationnel décrit par (4) ils ont obtenu [BATCHELOR et GREEN, 1972],

$$
\mu_{s}=\mu_{0}\left[1+\frac{5}{2} \phi+7.6 \phi^{2}+o\left(\phi^{2}\right)\right]
$$

Au fur et à mesure que l'on va vers des concentrations supérieures à $o\left(\phi^{2}\right)$, il n'est pas réaliste de s'attendre à des progrès analogues à ceux que l'on vient de présenter. Pour des concentrations où l'interaction entre les particules est dominante (par exemple $\phi>10 \%$ ), on s'est généralement tourné vers des modèles plus restrictifs (modèles cellulaires [SıMHA, 1952] par exemple) ou des

\footnotetext{
* Le coefficient de $\phi^{2}$ a été corrigé à 7.1 par KıM et Mifflin [1985].
}

corrélations des données viscométriques [RUTGERS, 1962]. A des concentrations très élevées, approchant du compactage maximum, il est encore possible d'effectuer quelques analyses utiles. FRANKEL et ACRIvOs [1967] ont utilisé la théorie de la lubrification pour prédire le comportement d'un modèle cellulaire d'une suspension concentrée et ils ont obtenu un bon accord avec quelques corrélations empiriques. Etant donné que, récemment, MARRUCCl et DENN [1985] ont trouvé quelques imperfections dans le choix des échelles utilisées par ces auteurs, on peut s'étonner du bon accord obtenu. Jusqu'à présent on n'a pas pu déceler les causes de cette apparente anomalie. De toute manière, des résultats rigoureux pour des suspensions concentrées de particules rigides ne paraissent pas accessibles par des méthodes similaires à celles utilisées dans le cas de forte dilution.

Etant donné les progrès réalisés pendant les cinq dernières années dans la conduite de calculs numériques complexes à un coût raisonnable, on est arrivé à un point où il est possible d'envisager de suivre, par application successive de l'équation de Stokes, la trajectoire des particules au travers de la suspension. Cette approche a été appelée « dynamique stokesienne " par analogie avec la dynamique moléculaire courante en chimie théorique. Par un choix judicieux de "pairwise additivity " BRADY et Bossis [1985] ont étudié certains phénomènes de transport dans les suspensions concentrées. Cette simulation numérique directe, quoique compliquée, semble être l'approche théorique la plus féconde quand la rhéologie est dominée par l'hydrodynamique.

Avant de quitter cette partie, revenons aux suspensions diluées et, en particulier, au problème mentionné précédemment de la "mémoire infinie " de l'orientation initiale des particules asymétriques. Quand les particules sont petites (ceci dans un sens qui sera précisé plus loin), le mouvement de rotation brownienne introduira des. orientations aléatoires qui auront tendance à effacer l'effet de l'orientation initiale. La prise en compte du mouvement brownien rend, bien entendu, les équations du mouvement plus compliquées. BURGERs [1938] reconnut ces problèmes et suggéra, il y a quelques années, un développement asymptotique. L'équation à résoudre, avant de calculer le comportement rhéologique, est celle de la fonction $f$ de distribution de probabilité de rotation.

$$
\frac{\partial f}{\partial t}+\nabla \cdot\left(f \dot{\mathbf{e}}_{h}\right)=D_{r} \nabla^{2} f
$$

où $\dot{\mathbf{e}}_{h}$ est la vitesse de rotation d'un vecteur d'orientation d'une particule, et $D_{r}$ est le coefficient de diffusion de rotation dû aux effets browniens.

LEAL et HINCH [1971] et HINCH et LEAL [1972, 1973] ont traité de manière détaillée le problème posé par Burgers dans le cas d'ellipsoïdes et sphéroïdes. Ils prolongent l'approche de Burgers et développent un traitement systématique des sphéroïdes pour des nombres de Péclet de rotation $P e_{r}=G / D_{r}$ petits et grands. Un exemple intéressant des résultats qui peuvent être obtenus est montré dans la figure 3 (page suiv.), où l'on observe l'évolution aléatoire du mouvement brownien au cours de la période transitoire pendant laquelle une suspension de sphéroïdes initialement orientés est sujette 


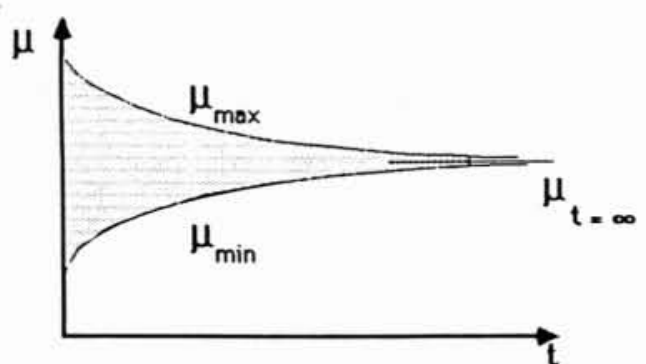

3. Evolution au cours du temps de la viscosité stationnaire d'une suspension de sphéroïdes, sous l'action du mouvement brownien.

à un cisaillement du type de l'équation (2). Des résultats sont aussi présentés montrant les contraintes de cisaillement et les contraintes normales en fonction du temps. Quand le nombre de Péclet augmente, l'effet du mouvement brownien de rotation diminue et la mémoire du fluide augmente.

Une indication que l'anisotropie induite par l'écoulement est importante même pour des grosses particules $(>100 \mu \mathrm{m})$ est donnée par les expériences de HUSBAND et GADALA-MARIA [1987]. Ils ont montré que la fonction de distribution de probabilité des couples n'est pas à symétrie sphérique quand l'état stationnaire est atteint, même si les particules sont sphériques et de densité égale à celle du fluide environnant.

Dans le cas des particules colloïdales, des forces autres qu'hydrodynamiques peuvent être importantes et même prédominantes. Nous nous sommes déjà penchés sur le mouvement brownien, qui introduit essentiellement une correction permettant d'utiliser les équations des milieux continus dans des situations physiques où la nature discontinue est évidente. Nous considérons ci-dessous des effets supplémentaires qui sont inhérents aux suspensions aqueuses de particules colloïdales.

\section{Complications introduites par la nature colloïdale des particules}

\section{Forces entre particules}

Quand on traite des suspensions de particules colloïdales hydrophobes dans un milieu aqueux, on est obligé de considérer deux sources supplémentaires de forces entre particules : dipôles induits et ions absorbés. Les premières ont été traditionnellement caractérisées par la constante de HAMAKER [1937] et sont de nature attractive. Des méthodes de calcul modernes [MAHANTY et NiNHAM, 1976], bien plus précises que l'approche d'Hamaker qui considère la combinaison linéaire des oscillations des dipôles, permettent d'estimer les forces induites entre ceux-ci, ou forces de London-van der Waals. Etant donné que nous sommes ici intéressés par les résultats qui découlent des forces de London-van der Waals et non par les méthodes qui permettent de les calculer, nos résultats s'inscrivent dans le cadre de ceux d'Hamaker.
Le second type de forces est produit par les ions absorbés à la surface des particules, donnant lieu à la double couche de Debye-Hückel. Il en résulte qu'un écran ionisé enveloppe chacune des particules et produit entre elles des forces de répulsion électrostatique. De même, nous nous satisferons des idées concernant l'épaisseur de la double couche, alors que la situation réelle est bien plus compliquée. La figure 4 montre schématiquement l'image que l'on se fait de la double couche électrique et représente la décroissance exponentielle de la concentration ionique. Les forces de London-van der Waals sont toujours prédominantes quand la distance entre particules est suffisamment grande ou petite. Comme le montre la figure 5, et suivant le rapport entre les forces d'attraction et de répulsion, il est possible d'obtenir des potentiels qui sont, soit purement attractifs, soit une combinaison d'attraction et de répulsion. Il faut noter que, pour une particule de rayon $a$, il existe un minimum secondaire dans la courbe de potentiel, dans le cas où $a \kappa=10^{*}$. Ceci ouvre la possibilité, pour les particules, de s'attirer mutuellement et de floculer dans ce minimum secondaire (pour des distances modérées entre elles). Par ailleurs, si les particules sont suffisamment proches, de manière à dépasser la barrière de potentiel au point $B$, elles vont s'agglomérer dans le minimum correspondant au point $A$. (A des distances très petites, les forces d'attraction dues aux dipôles, qui augmentent indéfiniment quand la distance diminue, sont contrecarrées par les forces de répulsion de Born qui tiennent compte du fait que les particules ne peuvent pas s'interpénétrer sans limite).

D'autres interactions, importantes pour la rhéologie des suspensions, sont celles causées par la présence dans le milieu aqueux ionisé d'additifs, généralement des polymères solubles, qui peuvent promouvoir ou inhiber la coagulation suivant des processus que l'on décrira plus loin. Pour l'instant, considérons l'influence, sur la structure colloïdale, des forces d'attraction de van der Waals et de répulsion électrostatique en présence d'un écoulement.

\section{Coagulation dynamique binaire}

Le comportement rhéologique des suspensions colloïdales dépendant, bien entendu, très fortement de l'état d'agglomération des particules, il est naturel de consacrer un paragraphe au processus dynamique d'agrégation en présence des forces hydrodynamiques et physico-chimiques. On considérera d'abord le cas où le mouvement brownien est négligeable. De plus, de manière à identifier les phénomènes essentiels qui contrôlent le processus, on étudiera plus particulièrement l'agrégation de deux particules sous l'influence d'un cisaillement.

En première approximation les courbes de potentiel de la figure 5 sont valables indépendamment du mouvement de la suspension. Donc, on doit s'attendre à ce qu'au repos les particules soient dans un état d'équilibre par rapport au potentiel et qu'elles soient ainsi localisées préférentiellement et donnent lieu à une structure donnée. Cependant, si l'on impose un écoulement de cisaillement de l'ensemble, des forces de nature hydrody-

$\left.{ }^{*}\right) \kappa^{-1}$ : longueur caractéristique de l'épaisseur de la double couche. 
namique viendront se superposer à celles déjà mentionnées. Dans un travail [ZEICHNER et SCHOWALTER, 1977] publié il y a quelques années, nous avons montré que le cisaillement pouvait stabiliser une dispersion en évitant la coagulation ou la déstabiliser en la provoquant. Ces résultats sont présentés d'une manière pratique dans la figure 6 , où l'on peut voir l'importance relative des forces de répulsion électrostatique, des forces d'attraction de London-van der Waals et des forces hydrodynamiques. En ce qui concerne les deux premières, leur rapport est donné par le «nombre de répulsion ",

$$
N_{R}=\frac{\varepsilon \psi_{0}^{2} a}{A}
$$

où $\varepsilon$ est la constante diélectrique, $\psi_{0}$ est le potentiel de surface (supposé constant sur la surface), et $A$ la constante de Hamaker. Pour les deux dernières on utilise le «nombre d'écoulement »

$$
N_{F}=\frac{6 \pi \mu a^{3} G}{A} .
$$

Pour l'exemple choisi, on voit que pour un nombre de répulsion un peu supérieur à 40 , la suspension aura tendance à floculer dans le minimum secondaire si le taux de cisaillement est suffisamment faible (Région II). Pour des taux de cisaillement plus élevés, les forces d'origine hydrodynamique sont suffisantes pour empêcher les particules de s'accumuler dans le minimum secondaire. Dans ce cas, la suspension peut rester stable (Région III), ou bien des particules peuvent franchir la barrière de potentiel, de sorte que la coagulation se produit dans le minimum primaire (Région I). Si le taux de cisaillement continue à augmenter, les forces d'origine hydrodynamique (lubrification) sont suffisantes pour éviter que l'on approche du minimum primaire. Une particule pénètre la zone d'influence d'une autre particule, sans s'approcher suffisamment pour être captée. Donc, la coagulation ne se produit pas, comme le montre la figure 6 pour des $N_{F}>5 \times 10^{3}$. Ceci est très important, du point de vue rhéologique, puisque cela indique que l'état d'agrégation, et donc le comportement (rhéologique), d'une suspension colloïdale peuvent être fortement influencés par l'intensité du cisaillement auquel est soumis l'échantillon. Notre intérêt pour la coagulation fut motivé principalement par le désir d'identifier les paramètres contrôlant les premières phases de la dynamique de la floculation et de développer ainsi la connaissance de la coagulation. Dans le cadre de cette présentation, nous nous intéressons aux structures qui résultent de la coagulation plutôt qu'aux détails du processus conduisant à leur formation.

Quoique, jusqu'à présent, nous ayons négligé le mouvement brownien, dans la plupart des suspensions colloïdales aqueuses il ne l'est point et, en particulier, il est prépondérant ou négligeable suivant que les diamètres des particules sont inférieurs à $0.1 \mu \mathrm{m}$ ou supérieurs à $10 \mu \mathrm{m}$ respectivement. Si les effets du mouvement brownien sont du même ordre de grandeur que ceux causés par les forces entre particules, les calculs deviennent très difficiles. Néanmoins, il y a des cas particuliers.

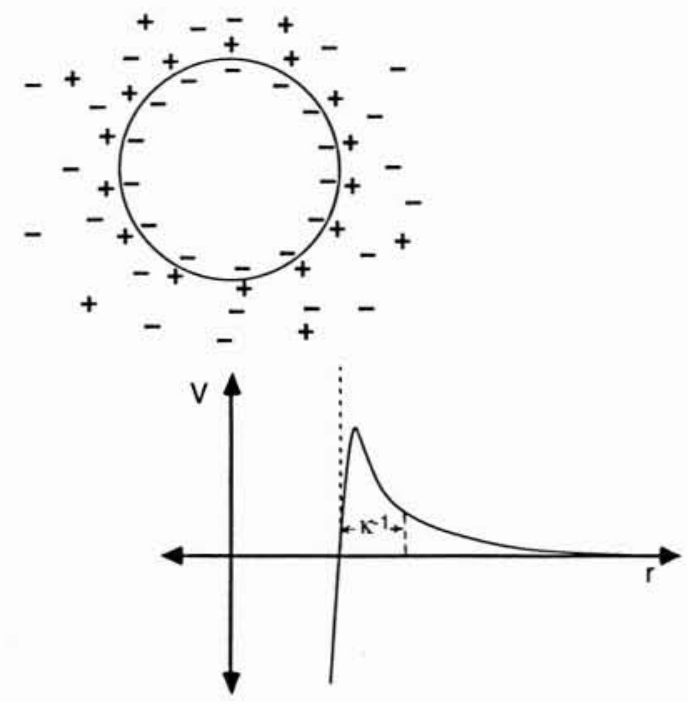

4. Evolution du potentiel électrostatique et de l'épaisseur de la double couche, caractérisé par la longueur $\mathrm{\kappa}^{-1}$.

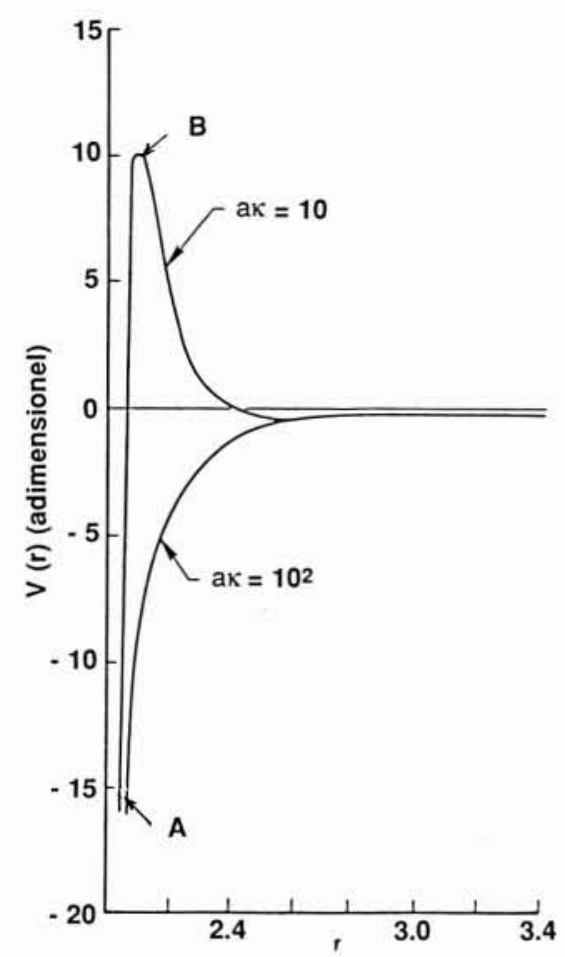

5. Courbes du potentiel interparticule dans le cas de double couche épaisse et double couche mince. (Rayon des particules sphériques $=a$.)

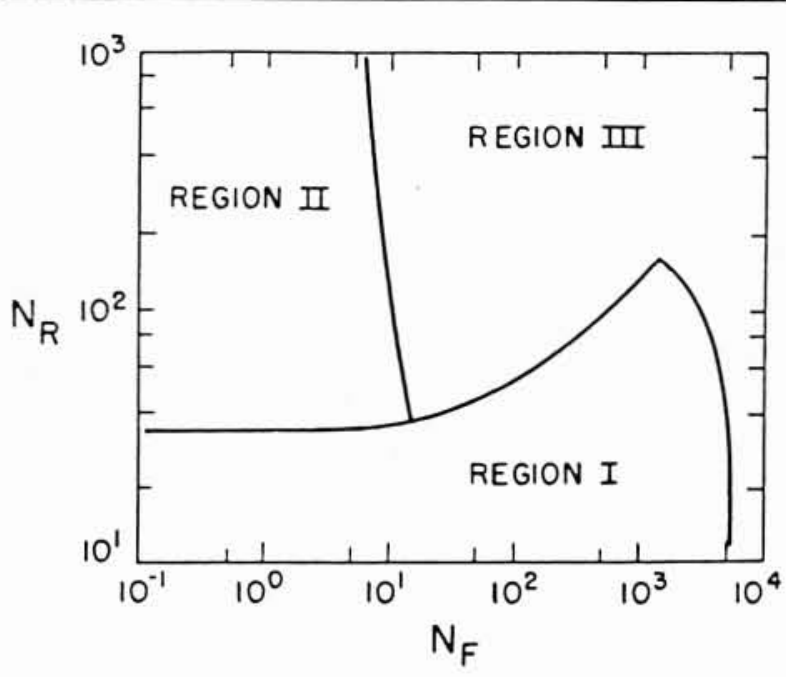

6. Plan de stabilité dans le cas d'un cisaillement simple. Région I. Coagulation dans un minimum primaire. Région II. Coagulation dans un minimum secondaire. Région III. Stabilité. 


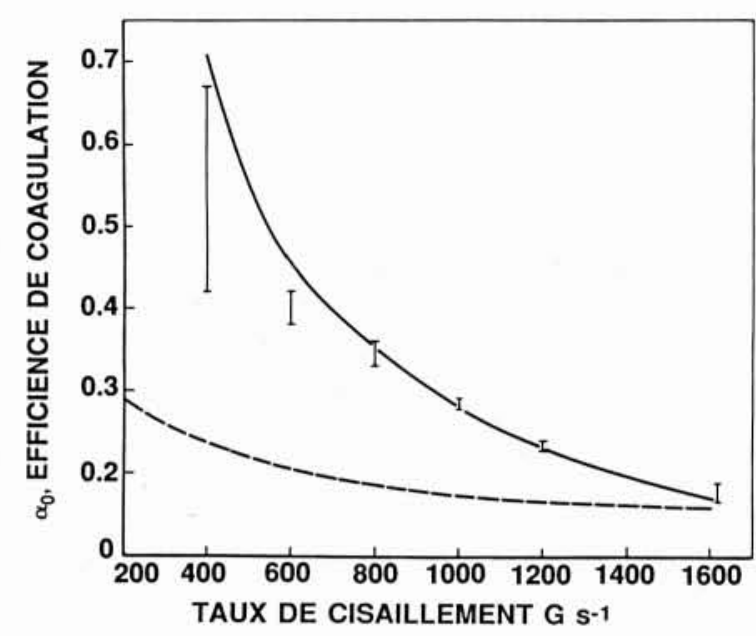

7. Influence du taux de cisaillement sur l'efficience de coagulation. --..-- : prédiction dans le cas $\mathrm{Pe}=\infty ;-$ : prédiction dans le cas $P e \gg 1$.

Les barres indiquent des résultats d'expériences [Feke et Schowalter, 1985] avec sphères de polystyrène $(a=0.33 \mu \mathrm{m}, 19<P e<150)$ dans un cisaillement simple. $\alpha_{0}$ est le rapport entre le taux de coagulation observé et celui si les particules cheminaient suivant les lignes de courant non perturbés d'un écoulement de cisaillement.

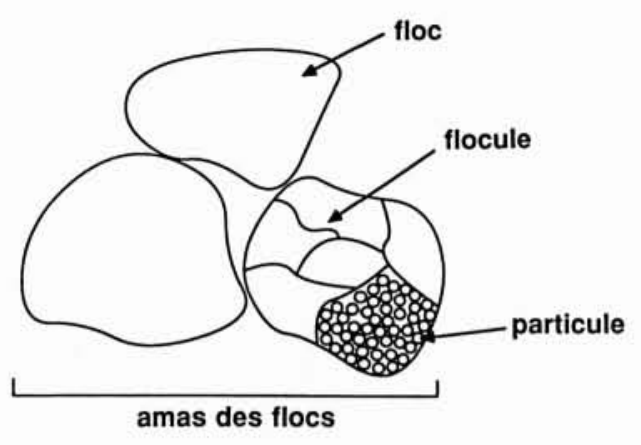

9. Structure d'une suspension agglomérée (d'après Hunter).

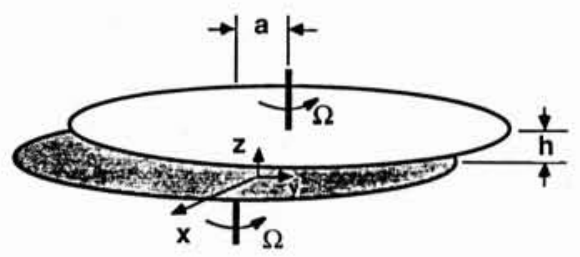

10. Diagramme schématique du rhéomètre de Maxwell.

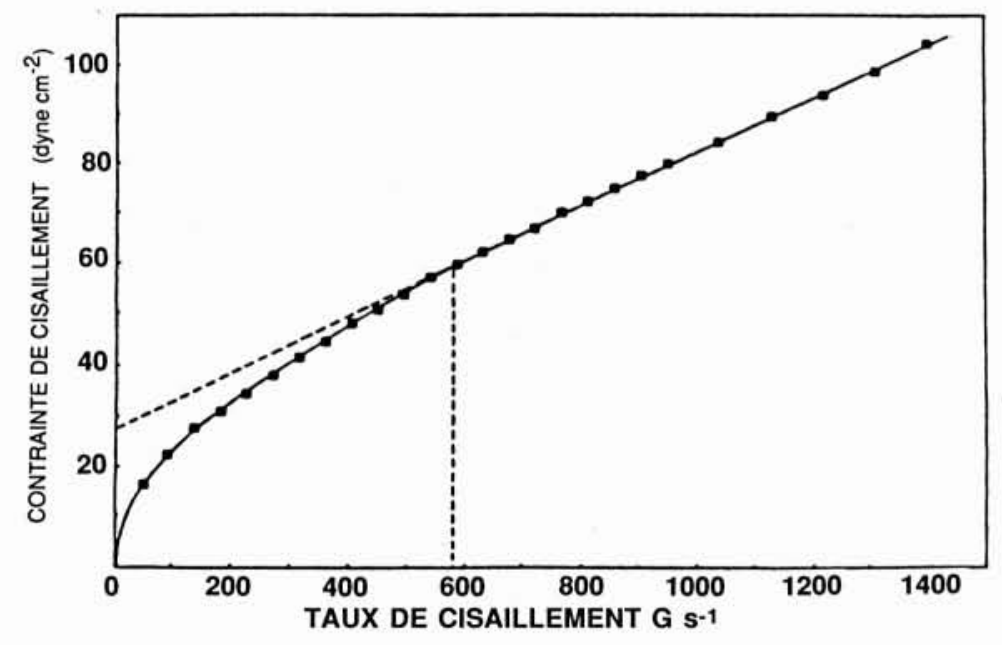

8. Rhéogramme typique des suspensions colloïdales modélisées par Hunter et collaborateurs [1982].

Par exemple, si les effets du mouvement brownien sont caractérisés par le nombre de Péclet, $P e=3 \pi \mu a^{3} G / k T$, où $T$ est la température absolue et $k$ la constante de Boltzmann, on peut montrer, pour des valeurs finies mais très grandes de celui-ci $(P e \gg 1)$ et pour un potentiel purement attractif, correspondant à une suspension instable, son influence sur le taux de coagulation. La figure 7 montre la modification de celui- 
ci quand le nombre de Péclet diminue à partir de l'infini [FEKE et SChOWALTER, 1985]. Ces résultats théoriques furent obtenus en développant, selon des puissances inverses de $P e$, les équations donnant la fonction de probabilité de distribution des couples et les équations de la trajectoire d'une particule par rapport à une autre.

Il est intéressant de connaître l'influence des résultats présentés ci-dessus sur la dynamique de la coagulation et la stabilité des suspensions collö̈dales. Bien que, dans le cadre de cette présentation, on se soit limité jusqu'à présent aux écoulements de cisaillement laminaires du type de ceux donnés par l'équation (2), il existe des calculs (mais pas de résultats expérimentaux) concernant un écoulement qui est l'analogue axisymétrique de l'équation (4): $\mathbf{v}=G[x,-y / 2,-z / 2]$. Bien que cet écoulement puisse paraître équivalent à l'équation (2) à chaque instant, il existe une différence fondamentale du fait de l'existence d'un rotationnel non nul dans le premier cas et de sa totale absence dans le second. Ceci a un effet remarquable sur les trajectoires des particules. Un exemple particulièrement frappant est celui des particules tournant les unes autour des autres dans un écoulement de cisaillement laminaire quand seules les forces d'interaction hydrodynamique sont prises en compte. Bien entendu, ces mouvements trouvent leur origine dans l'existence de lignes de courant fermées autour d'une particule isolée, comme il en a déjà été discuté précédemment. On peut s'attendre, et ceci est bien entendu confirmé, à ce que la tendance à la coagulation soit moindre dans le cas de l'écoulement élongationnel donné par (4) (ou l'analogue axisymétrique). Des courbes de stabilité similaires à celles de la figure 6 ont aussi été calculées dans le cas de l'élongation uniaxiale. Par ailleurs, les résultats sont pratiquement superposés si les échelles sont prises telles que, $N_{\text {Fis }}=2,75 N_{\text {Felong }}$ [ZEICHNER et SCHOWALTER, 1977].

\section{Dimensions des agglomérats et structure}

Aussi intéressants que puissent paraître les résultats sur la coagulation binaire, ils ne donnent qu'une indication sommaire sur les interactions entre particules et donc sur la rhéologie de la suspension. Des aspects importants restent en suspens, tels que par exemple, les structures associant plusieurs particules et la dimension et conformation de ces structures à l'équilibre quand la suspension est soumise à un écoulement donné. Par ailleurs, beaucoup de suspensions colloïdales d'intérêt industriel contiennent un ou plusieurs polymères dissous dans la phase continue.

Dans une série d'articles publiés dans les années 70 , Hunter et ses collaborateurs [FIRTH et HUNTER, 1976; VAN DE VEN et HUNTER, 1977 ; HUNTER, 1982] cherchèrent les paramètres qui auraient permis de corréler des données viscosimétriques pour des suspensions de particules rigides hydrophobes. Au moyen de quelques quantités, dont l'intérêt était considéré comme fondamental, ils proposèrent un modèle permettant de décrire le comportement quasi-binghamien (la figure 8 montre une courbe d'écoulement typique) de ces suspensions. Un aspect essentiel du modèle, et donc du succès de la corrélation, est que la suspension est tout d'abord soumise à un taux de cisaillement intense $\left(>2 \times 10^{3} \mathrm{~s}^{-1}\right)$ qui est ensuite réduit pour obtenir des courbes comme celle de la figure 8 . Il est supposé que le taux de cisaillement initial impose des caractéristiques structurelles qui persistent aux taux de cisaillement plus faibles. On crée ainsi des unités de base, appelées "flocules ", constituées d'un ensemble de particules colloïdales primaires et qui sont caractérisés par leur degré de compactation avec d'autres particules primaires. Celui-ci est déterminé par la quantité

$$
C_{F P}=\frac{\Phi_{F}}{\Phi_{P}}
$$

où $\Phi_{F}$ et $\Phi_{P}$ sont les fractions volumiques des « flocules " et des particules respectivement. Quand le taux de cisaillement est réduit, les "flocules " donnent lieu à la formation de flocs (avec le même $C_{F P}$ ) et, en dessous d'une valeur critique du taux de cisaillement, se forment des amas de flocs, figure 9. Ces derniers agrégats entrânent avec eux des quantités additionnelles de la phase liquide. D'après Hunter, les paramètres rhéologiques caractérisant la courbe de la figure 8 dépendent essentiellement de $C_{F P}$. Cette quantité est obtenue à partir d'un modèle simple de suspensions, comme celui d'Einstein, ou une modification de celui-ci pour des suspensions non diluées. Elle est inversement proportionnelle à la dimension des particules primaires et proportionnelle au carré du potentiel zeta $(\zeta)$. La plupart des résultats de Hunter, qui montrent un accord entre les données expérimentales et le modèle, sont présentés dans un article de synthèse [HUNTER, 1982].

Les détails de la méthode de Hunter ont été omis puisque l'objectif de cette présentation est de montrer les difficultés inhérentes au développement d'une théorie sur la rhéologie des colloïdes à partir du peu d'informations disponibles sur la façon dont les processus microscopiques se produisent. Il apparaît clairement que le développement d'une description théorique du comportement des phénomènes d'interaction entre particules et particules associées n'est pas à notre portée. Il est primordial, d'après nous, de constituer une banque de données crédible sur le comportement des flocs dans des situations pour lesquelles sont connus, raisonnablement bien, les paramètres hydrodynamiques et ceux du floc. A cette fin, nous avons mis en place un système d'écoulement, d'observation au microscope et d'acquisition de données que nous pensons tout à fait original [KOERNER, 1989]. Bien que les résultats obtenus soient encore préliminaires, il semble intéressant de décrire brièvement le principe de cet appareil. Il est basé sur le rhéomètre de Maxwell [MAXWELL et CHARTOFF, 1965] qui est constitué de deux plaques parallèles désaxées tournant à la même vitesse (fig. 10). Si les effets d'inertie sont négligeables, la cinématique de l'écoulement entre les plaques est donnée par [АВBOTT et WALTERS, 1970]

$$
\begin{aligned}
& E=\frac{\Omega \psi}{2}\left[\begin{array}{lll}
0 & 0 & 1 \\
0 & 0 & 0 \\
1 & 0 & 0
\end{array}\right] ; \\
& W=\Omega\left[\begin{array}{ccc}
0 & -1 & \psi / 2 \\
1 & 0 & 0 \\
-\psi / 2 & 0 & 0
\end{array}\right]
\end{aligned}
$$




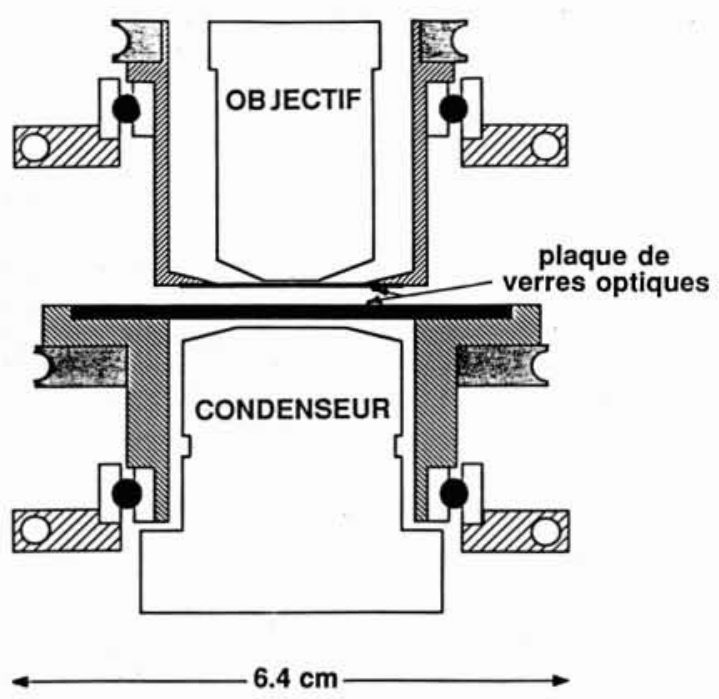

11. Intégration du microscope et des plaques qui produisent l'écoulement.

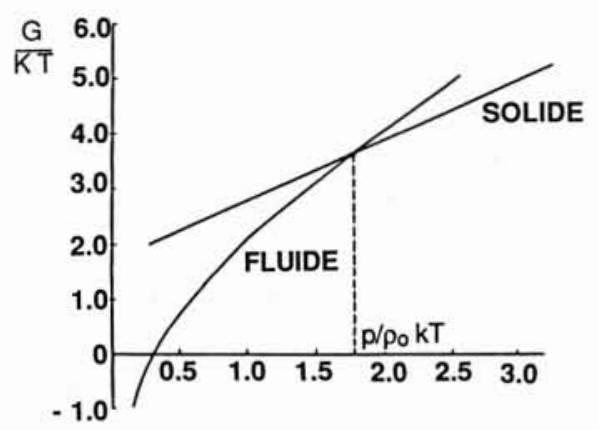

14. Energie libre (dans la forme adimensionnelle, $G / k T$ ) comme une fonction de la pression réduite $\left(p / \rho_{0} k T\right)$. Les résultats sont montrés pour la phase « solide " et la phase « liquide ", d'après Gast, et al. [1983]. La quantité $\rho_{0}$ indique la densité aux conditions de la compaction maximum ("closest packing $»), k=$ constante de Boltzmann, $T=$ température absolue. Les courbes correspondent à des valeurs constantes de la fraction volumique et du rapport $a / r_{g}$. Au point $p^{\prime} / \rho_{0} k T$, les deux phases peuvent coexister.

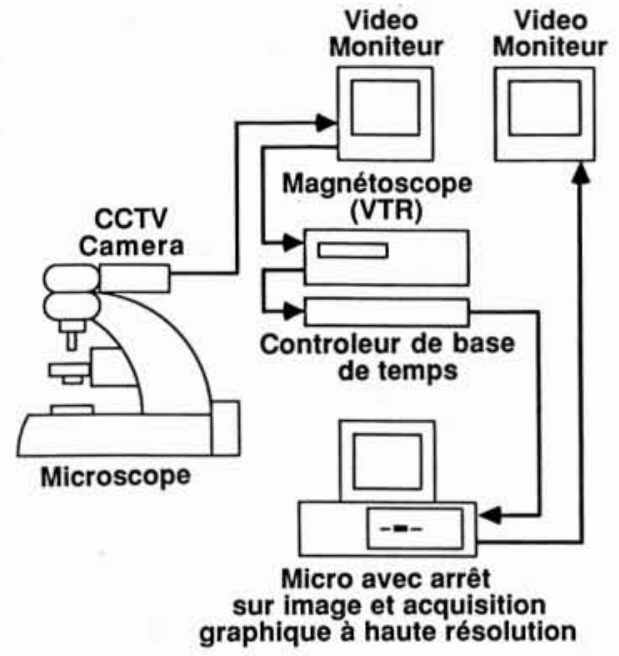

12. Diagramme schématique du système pour obtenir des données.

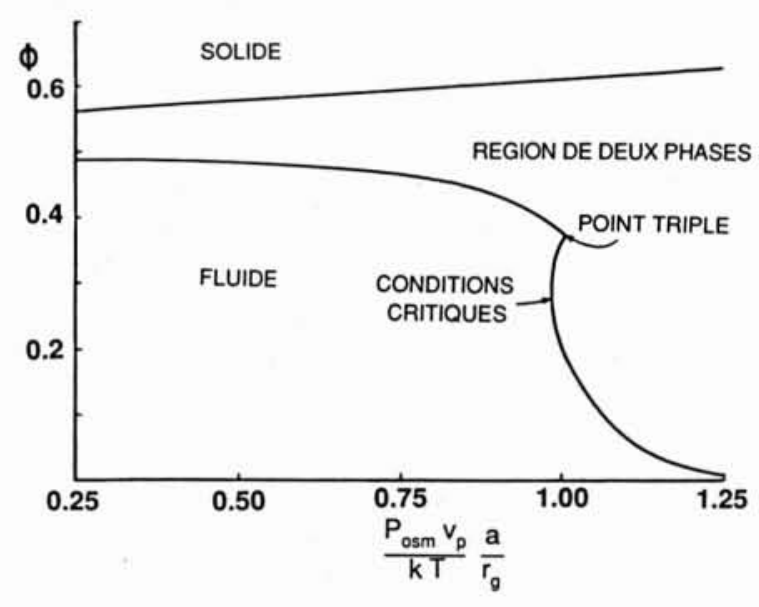

15. Diagramme de phase suivant la théorie de Gast et al. [1983]. $\phi=$ fraction volumique de colloïde, $p_{o s m}=$ pression osmotique, $v_{p}=$ volume du molécule polymère.

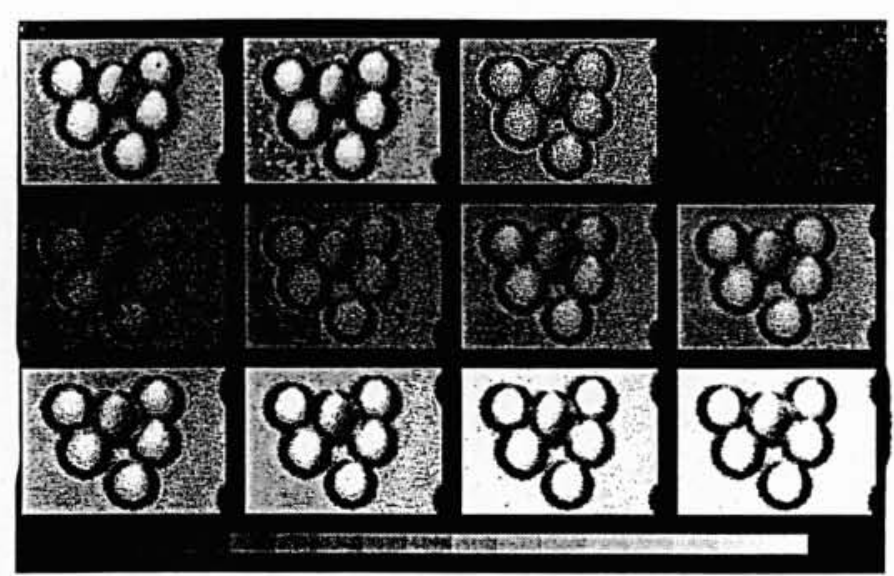

13. Visualisation d'un floc, d'après différentes options pour analyser l'image microscopique.

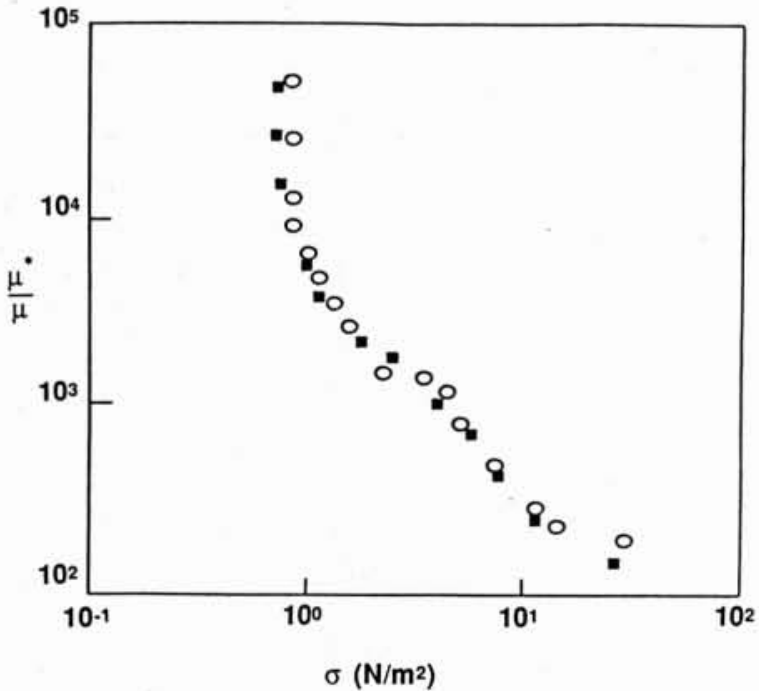

16. Comportement rhéologique de la phase solide pour des sphères de polystyrène dans l'eau avec du dextran. $\mu^{*} / \mu=$ viscosité réduite, $\sigma=$ contrainte de cisaillement. $O$ : résultats initiaux; $\bullet$ : répétition après $25 \mathrm{~min}$ [Patel, 1987]. 
où $E$ et $W$ sont les tenseurs du taux de déformation et du tourbillon respectivement, $\Omega$ est la vitesse de rotation et $\psi=a / h$ est l'excentricité (avec $a$. la distance entre les axes des plaques et $h$. l'entrefer). Dans notre cas les plaques sont constituées de verres optiques et le système est placé sous un microscope de sorte que, dans les conditions d'utilisation, les flocs soient visibles ( $f i g .11$ ). De manière à rendre possible l'exploitation des résultats, des enregistrements vidéo sont effectués au cours des expériences. Un diagramme schématique du montage est présenté dans la figure 12.

L'instrument est utilisé comme un outil dans lequel l'écoulement est contrôlé et les contraintes sont, idéalement, homogènes tandis que le taux de déformation et le tourbillon peuvent être modifiés indépendamment dans certaines limites. Nous avons utilisé les mêmes moyens, il y a quelque temps, pour étudier l'influence du cisaillement et de la rotation sur la déformation des gouttes [HAKImI et Schowalter, 1980]. Notre objectif actuel est de conduire des expériences similaires sur des flocs constitués de plusieurs particules primaires et d'observer directement ses dimensions et sa structure pour différentes valeurs du cisaillement et de la rotation. De manière à rendre le mouvement brownien négligeable, on opère avec des particules ayant approximativement $2 \mu \mathrm{m}$ de diamètre.

Les suspensions sont tout d'abord cisaillées de manière à obtenir un nombre suffisant de flocs dans les plages de dimension souhaitées. Dans notre cas, il s'agit d'obtenir approximativement l'agglomération d'une dizaine de particules primaires. Un échantillon dilué est ensuite introduit dans le rhéomètre.

L'objectif du travail expérimental est d'obtenir des informations concernant la dimension et la forme des flocs en termes statistiques et de les associer aux forces hydrodynamiques et de nature colloïdale qui s'exercent sur les particules. Donc, le traitement des données visuelles est tout-à-fait important et nous avons différentes idées sur la manière de calculer, emmagasiner et interpréter les données statistiques de nos expériences. Un exemple du type d'information qu'il est possible d'obtenir à partir de notre analyse d'images est donné dans la figure 13.

\section{Comportement des suspensions colloüdales en présence de polymères dissous}

Etant donné le comportement rhéologique des solutions de polymères, il est normal de s'attendre à ce que la rhéologie d'une suspension soit altérée par l'addition de faibles quantités de ces produits. L'effet le plus notable est probablement l'absorption des polymères à la surface des particules colloïdales, ce qui donne lieu à la formation d'une "peau " qui aura, évidemment, une influence considérable sur le phénomène de double couche abordé précédemment. De même, l'addition de polymères inhibe souvent la coagulation à travers le phénomène connu sous le nom de stabilisation stérique. La couche de polymère sur la surface empêche les particules de se trouver suffisamment proches pour pouvoir coaguler dans le minimum primaire de la courbe de potentiel. D'autres polymères sont connus par leur tendance à faire des pontages qui, au contraire, facilitent la coagulation.
Nous nous intéresserons ici aux effets dus aux modifications de comportement de la phase continue introduites par les polymères en ignorant les effets superficiels.

\section{Comportement à l'équilibre}

Un des aspects intéressants des polymères dissous est celui de la séparation des phases. On trouve que la suspension se divise en deux phases, avec différentes concentrations de particules qui peuvent coexister à l'état d'équilibre. Bien entendu, ceci se produit parce qu'il existe plus d'une concentration de particules pour une énergie libre donnée. Un élément important qui gouverne l'énergie libre est la pression osmotique due à la présence de polymères et le fait que, dans un lieu où les particules sont proches, la pression osmotique est différente de celle qui existe quand les polymères peuvent évoluer librement dans la phase continue en l'absence de particules. GAST, HALL et RUSSEL [1983] ont calculé l'énergie libre et les diagrammes de phase à partir d'un développement asymptotique du potentiel d'une sphère dure. Un échantillon de leurs résultats est présenté dans la figure 14. Ces derniers dépendent fortement du rapport entre la dimension des particules colloïdales (rayon : $a$ ), et celle du polymère, mesurée par son rayon de giration, $r_{g}$. La ligne en traits discontinus dans la figure (où $p=p^{\prime}$ ) correspond à une situation où la même énergie libre est présente dans un état de désordre (fluide) ou d'ordre (solide) et montre qu'une séparation de phase est possible. La réitération des calculs pour différentes phases volumiques permet d'obtenir un diagramme de phases comme celui de la figure 15. Il faut noter que, dans ce cas particulier, il est possible d'avoir deux phases fluides pour une certaine gamme de valeurs de l'abscisse. Bien que les frontières des domaines de phases aient des formes bien différentes de celles que l'on a habituellement pour des solides, liquides et gaz, l'interprétation du diagramme est tout-àfait classique. Les suspensions colloïdales dont la fraction volumique totale de particules correspond à un point situé dans la région à deux phases, se sépareront effectivement en deux "phases"; chacune d'elles contiendra des particules avec des fractions volumiques données par les frontières des domaines solide et liquide. La quantité relative de chaque phase est inversement proportionnelle à l'écart entre la fraction volumique totale et celle de la phase.

Plus récemment, Patel [1987], et PATEl et Russel [1987] ont effectué des expériences avec un système aqueux de polystyrène et de dextran pour vérifier les prédictions théoriques de Russel et de ses collaborateurs. Pour des valeurs de $a / r_{g}=6.9$, ils obtiennent un bon accord, tandis que pour $a / r_{g}=1.9$, c'est-à-dire pour des dimensions de particules proches de celles des macromolécules, il n'y a pas d'accord. L'objectif de Patel était d'étudier le comportement rhéologique de systèmes pouvant subir une transition de phase. En effet, la figure 16 montre bien que la phase la plus dense (celle dont la phase volumique est la plus élevée) a un comportement du type solide. Aux faibles taux de cisaillement $\left(\sigma \cong 1 \mathrm{~N} / \mathrm{m}^{2}\right)$, c'est bien le cas, mais la structure est détruite pour des taux de cisaillement suffisamment élevés $\left(\sigma>10 \mathrm{~N} / \mathrm{m}^{2}\right)$. Patel mesura aussi 
$G^{\prime}$ et $G^{\prime \prime}$ (composantes réelle et imaginaire, respectivement, du module complexe) et proposa une approche semi-empirique pour interpréter les données rhéologiques. C'est une théorie essentiellement basée sur le modèle proposé par ADLER et MILLS [1979], qui considèrent les flocs comme un milieu poreux à l'intérieur duquel l'équation de Brinkman est valable. Avec l'aide du critère de von Misès pour la rupture des flocs, il modélisa la destruction de la structure avec l'accroissement du cisaillement.

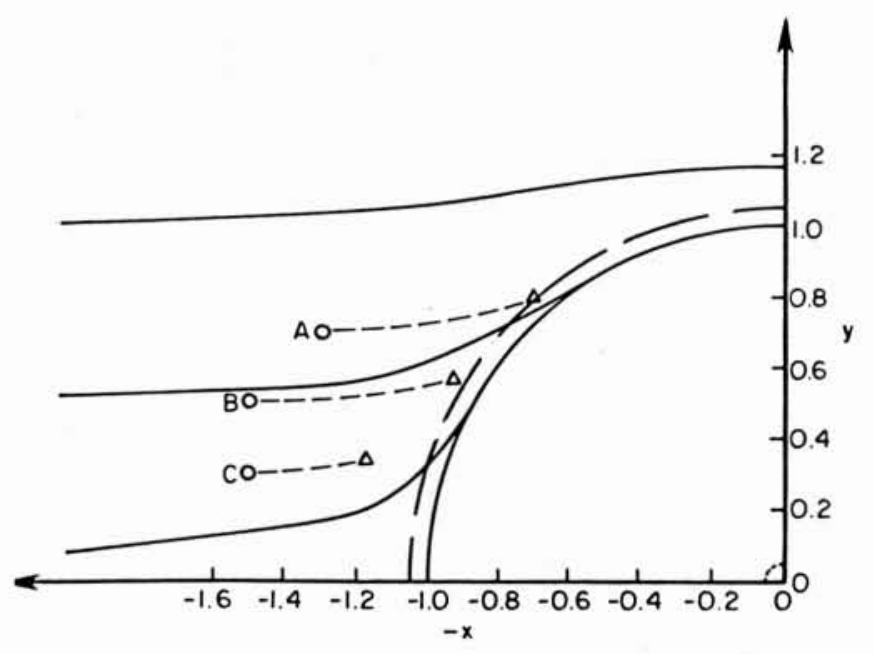

17. Trajectoires du centre d'une sphère, par rapport à celle de référence pour un milieu suspendant newtonien (trait plein) et non newtonien (We $=1.0$, trait discontinu $)$. Les triangles indiquent la fin du calcul. On doit faire une comparaison entre les pentes des traits newtoniens et non newtoniens.

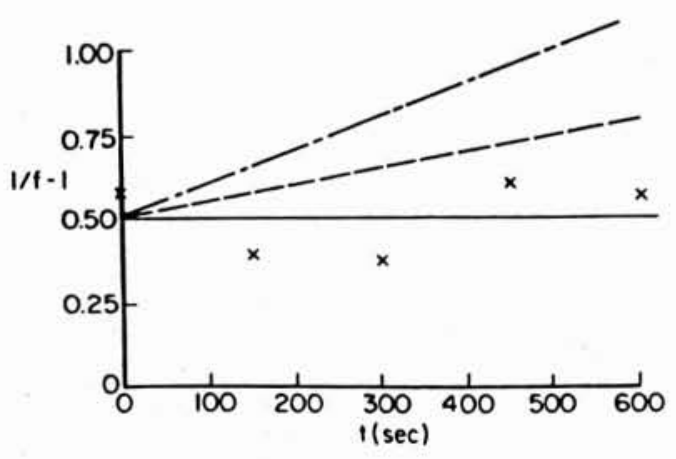

18. Disparition du nombre des particules isolées en fonction $d u$ temps. $f=$ fraction de particules isolées. Traits discontinus: résultats théoriques pour un fluide newtonien et deux barrières de potentiel. $\times$ et trait continu : données expérimentales dans une solution de HEC pour un nombre de Weissenberg $O(10)$.

\section{Comportement hors de l'équilibre}

Dans cet exposé on s'est surtout intéressé aux aspects dynamiques du comportement des suspensions colloïdales. Cependant la partie précédente a été consacrée au comportement à l'équilibre. Pendant un processus dynamique, il est fort possible que les particules ne se trouvent pas dans la sphère d'influence mutuelle pendant des temps suffisamment longs pour assurer la coagulation ou permettre le développement d'une structure. Dans les conditions où l'absorption ou la séparation des phases ne sont pas significatives, Mifflin et SCHOWALTER [1986, 1988] ont considéré théoriquement le problème de deux sphères placées dans un écoulement simple sans tenir compte des forces colloïdales usuelles. Le calcul permet d'apprécier l'influence de la fluidité et des contraintes normales. Ces dernières peuvent être prédominantes pour les particules très proches. On prédit alors une augmentation de la coagulation aux faibles taux de cisaillement mais une diminution aux forts taux du fait de la plus grande influence des contraintes normales. Ces conclusions sont obtenues en comparant les trajectoires de particules placées dans un écoulement de cisaillement simple d'un fluide obéissant à la loi corotationnelle de Jeffreys pour différentes valeurs du nombre de Weissenberg, We. Pour $W e=1$, les trajectoires montrent une séparation minimale plus petite (ce qui implique une plus grande tendance à la coagulation) que pour des fluides newtoniens. Cependant, pour $W e=10$, l'écart minimum entre particules augmente, ce qui correspond à une augmentation de la stabilité. Pour le plus petit $W e$, la figure 17 montre en traits continus les trajectoires des particules en fluide newtonien, et en traits discontinus celles en fluide non newtonien. On note que ces dernières sont moins rapidement éloignées de la sphère de référence que les premières. On a donc une tendance à la coagulation ou à la déstabilisation. Le contraire se produit pour $W e=10$. Des expériences conduites dans un écoulement de Couette avec des particules de latex de $600 \mathrm{~nm}$ dispersées dans une solution de HMC ont montré que, pour des nombres de Weissenberg estimés entre 10.5 et 15.3 , les taux de floculation étaient réduits d'un ordre de grandeur par rapport à la suspension sans addition de polymères ( $f i g$ 18).

Pour ce qui est des résultats présentés dans cette section, il est clair que l'addition de polymères à une suspension colloïdale a une influence double sur les paramètres rhéologiques. D'une part, les polymères confèrent des propriétés non newtoniennes à la phase continue et l'on doit au moins tenir compte des phénomènes hydrodynamiques non linéaires quand on considère un modèle de suspension colloïdale. D'autre part, l'état d'agrégation dépend fortement de la présence des polymères, même si leur concentration s'abaisse jusqu'à un pour cent.

\section{Quelques voies de recherche}

La présentation des recherches récentes ne prétend pas être exhaustive et ne s'est point limitée aux travaux les mieux connus. Les sujets abordés ont été choisis parce qu'ils font partie des préoccupations de l'auteur et parce qu'ils sont représentatifs des tendances des recherches 
actuelles. Ils peuvent donner l'impression que l'obtention de réponses pratiques rapides est hors de portée. Il est vrai que les études des colloïdes, et la rhéologie des colloïdes en particulier, dépend d'une variété de problèmes physiques et chimiques beaucoup plus large que d'autres sujets de recherche. Cependant, nous croyons que des études ponctuelles, comme celles présentées ici, peuvent être utiles à l'industrie. Elles peuvent définir des espaces de paramètres dans lesquels d'autres recherches sont nécessaires et peuvent aussi montrer l'existence de moyens à l'aide desquels la rhéologie des colloïdes industriels peut être modifiée. En même temps, des études fondamentales sur la rhéologie des suspensions colloïdales sont justifiées du fait de leur apport à notre connaissance du comportement des particules dans les écoulements.

Dans notre opinion, nous devons essayer de relier mieux qu'auparavant les résultats des modélisations numériques au comportement de la microstructure avec les observations directes. Du point de vue numérique, l'approche purement hydrodynamique de BRADY et Bossis [1985] peut être modifiée pour incorporer les forces physicochimiques. L'observation directe de l'accouplement de particules et la détermination des fonctions de distribution, telle que réalisée par GadalaMaria et ses collaborateurs (par exemple HUSBAND et GADALA-MARIA [1987]) fournit un maillon supplémentaire pour la compréhension du comportement des suspensions concentrées. On peut espérer, en étant réaliste, que le développement rapide des méthodes d'observation directe et l'amélioration des modélisations numériques vont rapprocher le moment où l'on pourra relier les caractéristiques macroscopiques des suspensions colloïdales aux processus dynamiques au niveau de leur microstructure.

\section{Remerciements}

La préparation de cet article n'aurait pas été possible sans l'aide d'un ensemble d'organismes, institutions et personnes physiques. Ceci comprend une invitation pour présenter un article de synthèse sur la rhéologie des suspensions colloïdales au $22^{\mathrm{e}}$ Congrès du Groupe français de rhéologie, l'aide financière de la National Science Foundation (Contrat CPE-8212327) pour l'étude de la dynamique de coagulation des colloïdes, une bourse de la John Simon Guggenheim Foundation et un poste de professeur invité à l'Ecole nationale supérieure de techniques avancées. Monsieur Daniel H. Fruman m'a apporté son aide dans la préparation de la version française de ce travail et Messieurs Daniel Huilier et Jean Briant ont contribué par des multiples commentaires et suggestions.

\section{Adresse de l'auteur :}

Professor William R. Schowalter, Department of Chemical Engineering, Princeton University, Princeton, NJ 08544, USA.

\section{Bibliographie}

AbBott, T. N. G., et K. WALters (1970), Rheometrical flow systems. Part 2. Theory for the orthogonal rheometer, including an exact solution of the Navier-Stokes equations, J. Fluid Mech., 40, 205.

Adler, P. M., et P. M. Mills (1979), Motion and rupture of a porous sphere in a linear flow field, J. Rheol., 23, 25.

Batchelor, G. K., et J. T. Green (1972), The determination of the bulk stress in a suspension of spherical particles to order $c^{2}$, J. Fluid Mech., 56, 401.

BrADY, J. et G. Bossis (1985), The rheology of concentrated suspensions of spheres in simple shear flow by numerical simulation, J. Fluid Mech, 155, 105.

Burgers, J. M. (1938) dans le «Second Report on Viscosity and Plasticity ", Verhandelingen der Koninkijke Nederlandsche Akademic van Wetenschappen, Eerste Sectic. Deel XV1, No. 4, pp. 113-184.

Cox, R. G., I. Y. Z. ZiA, et S. G. Mason (1968), Particle motions in sheared suspensions XXV. Streamlines around cylinders and spheres, J. Colloid and Interface Sci., 27, 7.

Einstein, A. (1906), A new determination of molecular dimensions, Ann. Physik, 19, 289.

Einstein, A. (1911), Corrections of my paper, «A new determination of molecular dimensions, Ann. Physik, 34, 591 .

FEkE, D. L., et W. R. Schowalter (1985), The influence of Brownian diffusion on binary flow-induced collision rates in colloidal dispersions, J. Colloid and Interface Sci., 106, 203.

FIRTH, B. A., et R. J. HunTER (1976), Flow properties of coagulated colloidal suspensions III. The elastic floc model, J. Colloid and Interface Sci., 57, 266.

Frankel, N. A., et A. Acrivos (1967), On the viscosity of a concentrated suspension of solid spheres, Chem. Eng. Sci., 22, 847.

FROHLICH, H., et R. SACK (1946), Theory of the rheological properties of dispersions, Proc. Roy. Soc., A185, 415.

Gast, A. P., C. K. Hall, et W. B. Russel (1983), Polymerinduced phase separations in nonaqueous colloidal suspensions, J. Colloid and Interface Sci., 96, 251.

Hakimi. F. S., et W. R. Schowalter (1980), The effects of shear and vorticity on deformation of a drop, J. Fluid Mech., 98, 635.

HAMAKER, H. C. (1937), The London-Van der Waals attraction between spherical particles, Physica 4, 1058.

HiNCH, E. J., et L. G. LEAL (1972), The effect of Brownian motion on the rheological properties of a suspension of non-spherical particles, J. Fluid Mech., 52, 683.

HiNCH, E. J., et L. G. LEAL (1973), Time-dependent shear flows of a suspension of particles with weak Brownian rotations, J. Fluid Mech., 57, 753.

HUNTER, R. J. (1982), The flow behavior of coagulated colloidal dispersions, Advances in Colloid and Interface Sci., 17, 197.

Husband, D. M., et F. Gadala-Maria (1987), Anisotropic particle distribution in dilute suspensions of solid spheres in cylindrical Couette flow, J. Rheol., 31, 95. 
JEFFERY, G. B. (1922), Motion of ellipsoidal particles immersed in a viscous fluid, Proc. Roy. Soc., A102, 161.

KıM, S., et R. T. Mifflin (1985), The resistance and mobility functions of two equal spheres in low-Reynolds-number flow, Phys. Fluids, 28, 2033.

Koerner, M. J. (1989), Ph. D. Thesis, Princeton University (en préparation).

LEAL, L. G., et E. J. HiNCH (1971), The effect of weak Brownian rotations on particles in shear flow, J. Fluid Mech., 46, 685.

Mahanty, J., et B. W. Ninham (1976), " Dispersion Forces ", Academic Press, New York.

Marrucci, G., et M. M. Denn (1985), On the viscosity of a concentrated suspension of solid spheres, Rheol. Acta, 24, 317.

Maxwell, B., et R. P. Chartoff (1965), Studies of a polymer melt in an orthogonal rheometer, Trans. Soc. Rheol., 9: 1,41 .

Mifflin, R. T., et W. R. Schowalter (1986), A numerical technique for three-dimensional steady flows of fluids of the memory-integral type, J. Non-Newtonian Fluid Mech., 20, 323.

Mifflin, R. T., et W. R. Schowalter (1988), Kinetics of the flocculation of colloids suspended in a viscoelastic fluid undergoing shear, AlChE J., 34, 1839.
OLDROYD, J. G. (1950), On the formulation of rheological equations of state, Proc. Roy. Soc., A200, 523.

PATEL, P. D. (1987), The rheological properties of aqueous colloidal suspensions phase separated by dissolved polymer, Ph. D. Dissertation, Princeton University.

PAtel, P. D., et W. B. Russel (1987), The rheology of polystyrene latices phase separated by dextran, J. Rheol., 31, 599.

Rallison, J. M. (1980), Note on the time-dependent deformation of a viscous drop which is almost spherical, J. Fluid Mech., 98, 625.

RUTGers, Ir., R. (1962), Relative viscosity of suspensions of rigid spheres in Newtonian liquids, Rheol. Acta, 2, 202, 305.

SimHA, Robert (1952), A treatment of the viscosity of concentrated suspensions, J. Appl. Phys., 23, 1020.

VAn de Ven, T. G. M., et R. J. Hunter (1977), The energy dissipation in sheared coagulated sols, Rheol. Acta, 16, 534.

Zeichner, G. R., et W. R. Schowalter (1977), Use of trajectory analysis to study stability of colloidal dispersions in flow fields, AlChE J., 23, 243.

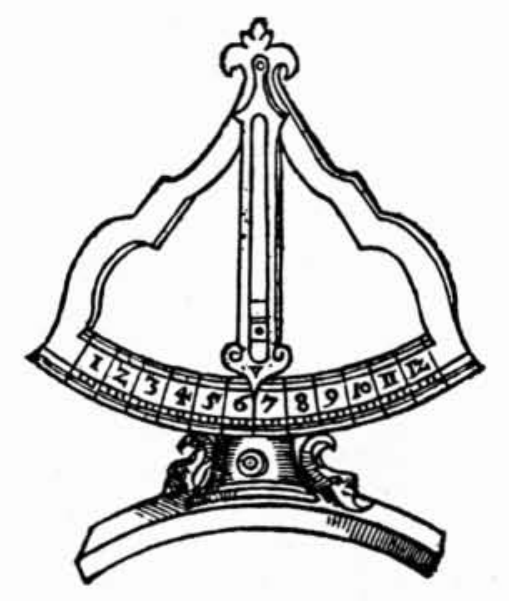

